\title{
Generating Functions for Certain Weighted Cranks
}

\author{
Shreejit Bandyopadhyay and Ae Ja Yee*
}

Dedicated to the memory of Srinivasa Ramanujan

\begin{abstract}
Recently, George Beck posed many interesting partition problems considering the number of ones in partitions. In this paper, we first consider the crank generating function weighted by the number of ones and obtain analytic formulas for this weighted crank function under conditions of the crank being less than or equal to some specific integer. We connect these cumulative and point crank functions to the generating functions of partitions with certain sizes of Durfee rectangles. We then consider a generalization of the crank for $k$-colored partitions, which was first introduced by Fu and Tang, and investigate the corresponding generating function for this crank weighted by the number of parts in the first subpartition of a $k$-colored partition. We show that the cumulative generating functions are the same as the generating functions for certain unimodal sequences.
\end{abstract}

Keywords. Partition crank, Weighted partitions, $k$-colored partitions, Unimodal sequences.

2010 Mathematics Subject Classification. 2010 AMS Classification Numbers: Primary, 11P81; Secondary, 05 A17.

\section{Introduction}

In the theory of partitions, one of the important combinatorial statistics is the crank, which was conjectured by Dyson for a combinatorial account for Ramanujan's famous mod 11 partition congruence in 1944 [Dys44] and discovered later by Andrews and Garvan [AnGa88]. Since the discovery, a great deal of research has been done on this statistic.

Recently, George Beck posed many interesting partition problems considering the number of ones in partitions. In this paper, we first consider the generating functions for the crank weighted by the number of ones. We then take a generalization of the crank for $k$-colored partitions, which was first introduced by Fu and Tang [FuTa17], and investigate the corresponding generating functions for this crank weighted by the number of parts in the first subpartition of a $k$-colored partition.

For a partition $\lambda$, let

$$
\begin{aligned}
& \omega(\lambda):=\text { number of parts of size } 1 \text { in } \lambda, \\
& \mu(\lambda):=\text { number of parts of } \lambda \text { greater than } \omega(\lambda) .
\end{aligned}
$$

Then, the crank is defined as

$$
\operatorname{crank}(\lambda):= \begin{cases}\lambda_{1}, & \text { if } \omega(\lambda)=0, \\ \mu(\lambda)-\omega(\lambda), & \text { if } \omega(\lambda) \geq 1,\end{cases}
$$

where $\lambda_{1}$ denotes the largest part of $\lambda$. We will also denote the number of parts of $\lambda$ by $\ell(\lambda)$ and the sum of parts by $|\lambda|$.

For $n \geq 1$ and any integer $m$, let $M(m, n)$ denote the number of partitions of $n$ with crank $m$. Also, we define $M(0,0)=M(1,1)=-M(0,1)=1$. In [AnGa88], Andrews and Garvan showed the following generating function formula:

$$
\sum_{n=0}^{\infty} \sum_{m=-\infty}^{\infty} M(m, n) z^{m} q^{n}=\frac{(q ; q)_{\infty}}{(z q ; q)_{\infty}(q / z ; q)_{\infty}},
$$

${ }^{*}$ The second author was partially supported by a grant (\#633963) from the Simons Foundation. We thank episciences.org for providing open access hosting of the electronic journal Hardy-Ramanujan Journal 
where, and in the sequel, we employ the following standard $q$-series notation:

$$
\begin{aligned}
(a ; q)_{0} & :=1 \\
(a ; q)_{n} & :=\prod_{k=0}^{n-1}\left(1-a q^{k}\right) \text { for } n \geq 1, \\
(a ; q)_{\infty} & :=\lim _{n \rightarrow \infty}(a ; q)_{n} .
\end{aligned}
$$

We now consider the generating function for partitions weighted by the crank and the number of ones:

$$
F(x, z, q):=\sum_{\lambda} x^{\omega(\lambda)} z^{\operatorname{crank}(\lambda)} q^{|\lambda|},
$$

where the sum is over all partitions $\lambda$.

Remark 1.1. It follows from $M(1,1)=-M(0,1)=M(-1,1)=1$ that the partition of 1 has three different crank values $0, \pm 1$. In the generating function in (1.2), the contributions of the partition of 1 with crank $1,0,-1$ are $z q,-q, x q / z$, respectively.

In [CCS, Eq. (3.1)], Chern showed that

$$
F(x, z, q)=\frac{(1-q)\left(x q^{2} ; q\right)_{\infty}}{(z q ; q)_{\infty}(x q / z ; q)_{\infty}} .
$$

The purpose of our paper is to first investigate the following point weighted crank function

$$
f_{j}(x, q)=\sum_{\operatorname{crank}(\lambda)=j} x^{\omega(\lambda)} q^{|\lambda|}
$$

and the corresponding cumulative function

$$
F_{j}(x, q)=\sum_{\operatorname{crank}(\lambda) \leq j} x^{\omega(\lambda)} q^{|\lambda|},
$$

where $j$ can be any integer.

Theorem 1.2. For $j \geq 1$,

$$
\begin{aligned}
f_{-j}(x, q) & =\sum_{n \geq 0} \frac{x^{n+j} q^{n^{2}+(j+2) n+j}}{(q ; q)_{n}\left(q^{2} ; q\right)_{n+j-1}} \\
f_{j}(x, q) & =\sum_{n \geq 0} \frac{x^{n} q^{n^{2}+(j+2) n+j}}{(q ; q)_{n}\left(q^{2} ; q\right)_{n+j-1}}
\end{aligned}
$$

and

$$
f_{0}(x, q)=(1-q) \sum_{n \geq 0} \frac{x^{n} q^{n^{2}+2 n}}{(q ; q)_{n}^{2}} .
$$

Theorem 1.3. For $j \geq 1$,

and

$$
\begin{aligned}
F_{-j}(x, q) & =\frac{(x q)^{j}}{1-x q} \sum_{n \geq 0} \frac{(x q)^{n} q^{n^{2}+n j}}{(q ; q)_{n}\left(q^{2} ; q\right)_{n+j-1}} \\
F_{j}(x, q) & =\frac{1}{1-x q} \sum_{n \geq 0} \frac{(x q)^{n} q^{n^{2}+n j}}{(q ; q)_{n}\left(q^{2} ; q\right)_{n+j-1}}
\end{aligned}
$$

$$
F_{0}(x, q)=\frac{1-q}{1-x q} \sum_{n \geq 0} \frac{(x q)^{n} q^{n^{2}}}{(q ; q)_{n}^{2}} .
$$


The $f_{0}(1, q)$ was first considered by Kaavya in [K11] and discussed by Huh and Kim in [HuKi21]. $F_{j}(1, q)$ was also studied by Hopkins, Sellers, Stanton, and Yee in [HS20], [HSY22], and [HSY].

The notion of partitions can be generalized to $k$-colored partitions for $k \geq 2$. A $k$-colored partition $\pi$ of $n$ is a $k$-tuple of partitions $\pi=\left(\pi_{1}, \pi_{2}, \ldots, \pi_{k}\right)$ with $|\pi|:=\left|\pi_{1}\right|+\left|\pi_{2}\right|+\cdots+\left|\pi_{k}\right|=n$. Let $p_{k}(n)$ denote the number of $k$-colored partitions of $n$. Clearly, the following is its generating function:

$$
\sum_{n=0}^{\infty} p_{k}(n) q^{n}=\frac{1}{(q ; q)_{\infty}^{k}}
$$

In [FuTa17], Fu and Tang introduced a generalized crank for $k$-colored partitions, which is defined by

$$
\operatorname{crank}_{k}(\pi)=\ell\left(\pi_{1}\right)-\ell\left(\pi_{2}\right) .
$$

This generalized crank for $k$-colored partitions is called the $k$-crank. Let $M_{k}(m, n)$ denote the number of $k$-colored partitions of $n$ with $k$-crank $m$. Then, we can easily see from the definition that

$$
\sum_{n=0}^{\infty} \sum_{m=-\infty}^{\infty} M_{k}(m, n) z^{m} q^{n}=\frac{(q ; q)_{\infty}^{2-k}}{(z q ; q)_{\infty}(q / z ; q)_{\infty}}
$$

Let

$$
G_{k}(x, z, q):=\sum_{\pi} x^{\ell\left(\pi_{1}\right)} z^{\operatorname{crank}_{k}(\pi)} q^{|\pi|},
$$

where the sum is over all $k$-colored partitions $\pi$. Then, we get

$$
G_{k}(x, z, q)=\frac{(q ; q)_{\infty}^{2-k}}{(x z q ; q)_{\infty}(q / z ; q)_{\infty}} .
$$

The second purpose of this paper is to investigate the corresponding cumulative function

$$
G_{k, d}(x, q)=\sum_{\operatorname{crank}_{k}(\pi) \geq d} x^{\ell\left(\pi_{1}\right)} q^{|\pi|}
$$

where $d$ can be any integer.

Theorem 1.4. For $k \geq 2$ and $d \geq 0$,

$$
G_{k, d}(x, q)=\frac{1}{(q ; q)_{\infty}^{k-2}} \sum_{n \geq 0} \frac{(x q)^{n+d}}{(q ; q)_{n+d}(q ; q)_{n}},
$$

and

$$
G_{k,-d}(x, q)=\frac{1}{(q ; q)_{\infty}^{k-2}} \sum_{n \geq 0} \frac{(x q)^{n}}{(q ; q)_{n+d}(q ; q)_{n}} .
$$

The rest of this paper is organized as follows. In Section 2, the weighted crank generating functions $f_{j}(x, q)$ and $F_{j}(x, q)$ will be studied, and the proofs of Theorems 1.2 and 1.3 will be given. In Section 3 , the weighted $k$-crank generating function $G_{k, d}(x, q)$ will be studied with the proof of Theorem 1.4 given. A connection of this generating function to stacks with summits will be shown as well. This paper will end with a short concluding remark in Section 4. 


\section{The Crank of Partitions Weighted by the Number of Ones}

In this section, we consider with the crank of a partition weighted by the number of ones occurring in the partition. We first note the following theorem, which was first proved in [Che21, Eq. 3.8], and can also be found in [Ban]. In order to make this paper self-contained, we provide its proof.

Theorem 2.1. We have

$$
\frac{(x q ; q)_{\infty}}{(z q ; q)_{\infty}(x q / z ; q)_{\infty}}=\frac{1}{(q ; q)_{\infty}} \sum_{n \geq 1}(-1)^{n-1} q^{\left(\begin{array}{c}
n \\
2
\end{array}\right)} \frac{(x q ; q)_{n}}{(q ; q)_{n-1}}\left(\frac{1}{1-z q^{n}}+\frac{x q^{n} / z}{1-x q^{n} / z}\right) .
$$

Proof. We have the following identity by [GaRa04, Eq.(2.7.1)]:

$$
\sum_{n \geq 0} \frac{\left(1-a q^{2 n}\right)(a ; q)_{n}(b ; q)_{n}(c ; q)_{n}(d ; q)_{n}}{(1-a)(q ; q)_{n}(a q / b ; q)_{n}(a q / c ; q)_{n}(a q / d ; q)_{n}}\left(\frac{a q}{b c d}\right)^{n}=\frac{(a q ; q)_{\infty}\left(\frac{a q}{b c} ; q\right)_{\infty}\left(\frac{a q}{b d} ; q\right)_{\infty}\left(\frac{a q}{c d} ; q\right)_{\infty}}{\left(\frac{a q}{b} ; q\right)_{\infty}\left(\frac{a q}{c} ; q\right)_{\infty}\left(\frac{a q}{d} ; q\right)_{\infty}\left(\frac{a q}{b c d} ; q\right)_{\infty}}
$$

Set $a=x, b=x / z, c=z, d \rightarrow \infty$ in (2.8), which reduces to

$$
\sum_{n \geq 0}(-1)^{n} q^{\left(\begin{array}{c}
n+1 \\
2
\end{array}\right)} \frac{(x ; q)_{n}\left(1-x q^{2 n}\right)}{(q ; q)_{n}(1-x)} \frac{(1-x / z)(1-z)}{\left(1-z q^{n}\right)\left(1-x q^{n} / z\right)}=\frac{(x q ; q)_{\infty}(q ; q)_{\infty}}{(z q ; q)_{\infty}(x q / z ; q)_{\infty}} .
$$

Noticing

$$
\frac{(1-z)(1-x / z)\left(1-x q^{2 n}\right)}{\left(1-z q^{n}\right)\left(1-x q^{n} / z\right)}=-\left(1-q^{n}\right)\left(1-x q^{n}\right)\left(\frac{1}{q^{n}\left(1-z q^{n}\right)}+\frac{x / z}{\left(1-x q^{n} / z\right)}\right)+\left(\frac{1-x q^{2 n}}{q^{n}}\right),
$$

we see that the left hand side of (2.9) becomes

$$
\sum_{n \geq 0} \frac{(-1)^{n-1} q^{\left(\begin{array}{c}
n \\
2
\end{array}\right)}(x q ; q)_{n}\left(1-q^{n}\right)}{(q ; q)_{n}}\left(\frac{1}{1-z q^{n}}+\frac{x q^{n} / z}{1-x q^{n} / z}\right)+\sum_{n \geq 0} \frac{(-1)^{n} q^{\left(\begin{array}{c}
n \\
2
\end{array}\right)(x ; q)_{n}\left(1-x q^{2 n}\right)}}{(1-x)(q ; q)_{n}} .
$$

We next note that the second sum in (2.10) vanishes. Indeed, if we set $a=x, b c=q x, d \rightarrow \infty$ in (2.8), we find that

$$
\sum_{n \geq 0} \frac{(-1)^{n} q^{\left(\begin{array}{c}
n \\
2
\end{array}\right)}(x ; q)_{n}\left(1-x q^{2 n}\right)}{(1-x)(q ; q)_{n}}=\frac{(x q ; q)_{\infty}(1 ; q)_{\infty}}{\left(\frac{x q}{b} ; q\right)_{\infty}(b ; q)_{\infty}}=0 .
$$

Thus, by (2.9) and (2.10), we have

$$
\sum_{n \geq 1} \frac{(-1)^{n-1} q^{\left(\begin{array}{c}
n \\
2
\end{array}\right)}(x q ; q)_{n}}{(q ; q)_{n-1}}\left(\frac{1}{1-z q^{n}}+\frac{x q^{n} / z}{1-x q^{n} / z}\right)=\frac{(x q ; q)_{\infty}(q ; q)_{\infty}}{(z q ; q)_{\infty}(x q / z ; q)_{\infty}}
$$

where the summation on the left hand side starts from $n=1$ because the $n=0$ term becomes 0 . The theorem now follows on dividing both sides by $(q ; q)_{\infty}$.

We also need the following identity of Fine from [Fin88, (20.51)].

Lemma 2.2. We have

$$
\frac{1}{(b q ; q)_{\infty}} \sum_{n \geq 0}(-1)^{n} b^{n} q^{\left(\begin{array}{c}
n+1 \\
2
\end{array}\right)} \frac{(t ; q)_{n}}{(q ; q)_{n}}=\sum_{n \geq 0} \frac{(b t)^{n} q^{n^{2}}}{(q ; q)_{n}(b q ; q)_{n}} .
$$

Using (1.3) and (2.7), we get the following lemma, which can also be found in [Che21, Eq. 3.9].

Lemma 2.3. We have

$$
F(x, z, q)=\frac{1}{(1-x q)\left(q^{2} ; q\right)_{\infty}} \sum_{n \geq 1}(-1)^{n-1} q^{\left(\begin{array}{c}
n \\
2
\end{array}\right)} \frac{(x q ; q)_{n}}{(q ; q)_{n-1}}\left(\frac{1}{1-z q^{n}}+\frac{x q^{n} / z}{1-x q^{n} / z}\right) .
$$




\section{A. Proof of Theorem 1.2}

In this section, we prove the formulas for the point weighted generating function $f_{j}(x, q)$ both analytically and combinatorially.

Analytic Proof. Suppose $j \geq 1$. By Lemma 2.3,

$$
\begin{aligned}
f_{-j}(x, q) & =\frac{1}{(1-x q)\left(q^{2} ; q\right)_{\infty}} \sum_{n \geq 1}(-1)^{n-1} q^{n(n-1) / 2} \frac{(x q ; q)_{n}}{(q ; q)_{n-1}} x^{j} q^{n j} \\
& =\frac{1}{(1-x q)\left(q^{2} ; q\right)_{\infty}} \sum_{n \geq 0}(-1)^{n} q^{n(n+1) / 2} \frac{(x q ; q)_{n+1}}{(q ; q)_{n}} x^{j} q^{(n+1) j} \\
& =\frac{(x q)^{j}}{\left(q^{2} ; q\right)_{\infty}} \sum_{n \geq 0}(-1)^{n} q^{n(n+1) / 2} \frac{\left(x q^{2} ; q\right)_{n}}{(q ; q)_{n}} q^{n j} \\
& =\frac{(x q)^{j}\left(q^{j+1} ; q\right)_{\infty}}{\left(q^{2} ; q\right)_{\infty}} \sum_{n \geq 0} \frac{q^{n^{2}}\left(x q^{j+2}\right)^{n}}{(q ; q)_{n}\left(q^{j+1} ; q\right)_{n}} \\
& =\sum_{n \geq 0} \frac{x^{n+j} q^{n^{2}+(j+2) n+j}}{(q ; q)_{n}\left(q^{2} ; q\right)_{n+j-1}}
\end{aligned}
$$

where the second to last equality follows by setting $b=q^{j}, t=x q^{2}$ in Lemma 2.2, proving the formula for $f_{-j}(x, q)$.

Similarly,

$$
f_{j}(x, q)=\frac{q^{j}}{\left(q^{2} ; q\right)_{\infty}} \sum_{n \geq 0}(-1)^{n} q^{n(n+1) / 2} \frac{\left(x q^{2} ; q\right)_{n}}{(q ; q)_{n}} q^{n j}
$$

and thus by setting $b=q^{j}, t=x q^{2}$ in Lemma 2.2 , we get

$$
f_{j}(x, q)=\sum_{n \geq 0} \frac{x^{n} q^{n^{2}+(j+2) n+j}}{(q ; q)_{n}\left(q^{2} ; q\right)_{n+j-1}},
$$

proving the formula for $f_{j}(x, q)$ as before.

Again, by Lemma 2.2 and with some simplifications, we get

$$
f_{0}(x, q)=\frac{1}{\left(q^{2} ; q\right)_{\infty}} \sum_{n \geq 0}(-1)^{n} q^{n(n+1) / 2} \frac{\left(x q^{2} ; q\right)_{n}}{(q ; q)_{n}} .
$$

Setting $b=1, t=x q^{2}$ in Lemma 2.2 , we can similarly verify the formula for $f_{0}(x, q)$.

Combinatorial Proof. We prove Theorem 1.2 combinatorially. Suppose $\lambda$ is a partition with crank $-j$ for some $j \geq 1$. Then $\omega(\lambda) \neq 0$. If $\mu(\lambda)=n$, then by the definition of crank, $\omega(\lambda)=n+j$. So $\lambda$ has in its Ferrers diagram an $n+j$ block of ones, an $n \times(n+j+1)$ rectangle and two other blocks, one having parts at least 2 and at most $n+j$, and the other having at most $n$ parts. Thus, $\lambda$ can be generated by

$$
\sum_{n \geq 0} \frac{(x q)^{n+j} q^{n(n+j+1)}}{(q ; q)_{n}\left(q^{2} ; q\right)_{n+j-1}}
$$

which verifies the formula for $f_{-j}(x, q)$ combinatorially.

Next, suppose the crank of a partition $\lambda$ is $j$ for some $j \geq 1$. If $\omega(\lambda)=n>0$, then $\mu(\lambda)$ must be $n+j$. So there are $n+j$ parts greater than $n$, and hence the Ferrers diagram of $\lambda$ has $n$ ones, an 
$(n+j) \times(n+1)$ rectangle and two other blocks, one having parts at least 2 and at most $n$, and the other having at most $n+j$ parts. Thus, $\lambda$ can be generated by

$$
\sum_{n \geq 1} \frac{(x q)^{n} q^{(n+1)(n+j)}}{\left(q^{2} ; q\right)_{n-1}(q ; q)_{n+j}} .
$$

If $\omega(\lambda)=0$, then $\lambda_{1}=j$, so the generating function is

$$
\frac{q^{j}}{\left(q^{2} ; q\right)_{j-1}}
$$

Adding this generating function for $\omega(\lambda)=0$ to (2.11), we have verified the formula for $f_{j}(x, q)$ for $j \geq 1$.

Lastly, if the crank is zero, then with $\omega(\lambda)=n>0$, we have $n$ parts of size at least $n+1$ and hence the generating function is

$$
\sum_{n \geq 1} \frac{(x q)^{n} q^{n(n+1)}}{(q ; q)_{n}\left(q^{2} ; q\right)_{n-1}} .
$$

If $\omega(\lambda)=0$, then we see from Remark 1.1 that $\lambda$ is the empty partition or the partition of 1 with crank 0 , and their contributions to $F(x, z, q)$ are $1-q$. Thus, the full generating function for crank zero is

$$
(1-q) \sum_{n \geq 0} \frac{x^{n} q^{n^{2}+2 n}}{(q ; q)_{n}^{2}}
$$

as desired.

\section{B. Proof of Theorem 1.3}

We now consider the cumulative generating function $F_{j}(x, q)$. We will first prove this theorem analytically using Lemmas 2.2 and 2.3, and then prove it combinatorially using the concept of Durfee rectangles.

Analytic Proof. For $j \geq 1$, we find by Lemma 2.3 that

$$
\begin{aligned}
F_{-j}(x, q) & =\frac{1}{(1-x q)\left(q^{2} ; q\right)_{\infty}} \sum_{n \geq 1}(-1)^{n-1} q^{n(n-1) / 2} \frac{(x q ; q)_{n}}{(q ; q)_{n-1}} \frac{x^{j} q^{n j}}{1-x q^{n}} \\
& =\frac{(x q)^{j}}{(1-x q)\left(q^{2} ; q\right)_{\infty}} \sum_{n \geq 0}(-1)^{n} q^{n j+n(n+1) / 2} \frac{(x q ; q)_{n}}{(q ; q)_{n}} \\
& =\frac{(x q)^{j}\left(q^{j+1} ; q\right)_{\infty}}{(1-x q)\left(q^{2} ; q\right)_{\infty}} \sum_{n \geq 0} \frac{\left(x q^{j+1}\right)^{n} q^{n^{2}}}{(q ; q)_{n}\left(q^{j+1} ; q\right)_{n}} \\
& =\frac{(x q)^{j}}{(1-x q)} \sum_{n \geq 0} \frac{(x q)^{n} q^{n^{2}+n j}}{(q ; q)_{n}\left(q^{2} ; q\right)_{n+j-1}}
\end{aligned}
$$

where the penultimate step follows on setting $b=q^{j}, t=x q$ in Lemma 2.2. Hence we have the formula for $F_{-j}(x, q)$. 
Next, we check $F_{0}(x, q)$. By Lemma 2.3 , we get

$$
\begin{aligned}
F_{0}(x, q) & =\frac{1}{(1-x q)\left(q^{2} ; q\right)_{\infty}} \sum_{n \geq 1}(-1)^{n-1} q^{n(n-1) / 2} \frac{(x q ; q)_{n}}{(q ; q)_{n-1}}\left(1+\frac{x q^{n}}{1-x q^{n}}\right) \\
& =\frac{1}{(1-x q)\left(q^{2} ; q\right)_{\infty}} \sum_{n \geq 0}(-1)^{n} q^{n(n+1) / 2} \frac{(x q ; q)_{n}}{(q ; q)_{n}} \\
& =\frac{1-q}{(1-x q)} \sum_{n \geq 0} \frac{(x q)^{n} q^{n^{2}}}{(q ; q)_{n}^{2}},
\end{aligned}
$$

where the last equality follows from Lemma 2.2 by setting $b=1$ and $t=x q$.

Now, for $j \geq 1$, again by Lemma 2.3,

$$
\sum_{n \geq 0} \sum_{\substack{\lambda \vdash n \\ \operatorname{crank}(\lambda)>j}} x^{\omega(\lambda)} q^{n}=-\frac{1}{(1-x q)\left(q^{2} ; q\right)_{\infty}} \sum_{n \geq 1}(-1)^{n} q^{n(n+1) / 2+n j} \frac{(x q ; q)_{n}}{(q ; q)_{n}} .
$$

Set $b=q^{j}, t=x q$ in Lemma 2.2 to get

$$
\sum_{n \geq 0}(-1)^{n} q^{n(n+1) / 2+n j} \frac{(x q ; q)_{n}}{(q ; q)_{n}}=\left(q^{j+1} ; q\right)_{\infty} \sum_{n \geq 0} \frac{\left(x q^{j+1}\right)^{n} q^{n^{2}}}{(q ; q)_{n}\left(q^{j+1} ; q\right)_{n}} .
$$

Hence,

$$
\sum_{n \geq 1}(-1)^{n} q^{n(n+1) / 2+n j} \frac{(x q ; q)_{n}}{(q ; q)_{n}}=\left(q^{j+1} ; q\right)_{\infty} \sum_{n \geq 0} \frac{\left(x q^{j+1}\right)^{n} q^{n^{2}}}{(q ; q)_{n}\left(q^{j+1} ; q\right)_{n}}-1
$$

so that

$$
\begin{aligned}
\sum_{n \geq 0} \sum_{\substack{\lambda \vdash n \\
\operatorname{cank}(\lambda)>j}} x^{\omega(\lambda)} q^{n} & =\frac{1}{(1-x q)\left(q^{2} ; q\right)_{\infty}}\left[1-\left(q^{j+1}\right)_{\infty} \sum_{n \geq 0} \frac{\left(x q^{j+1}\right)^{n} q^{n^{2}}}{(q ; q)_{n}\left(q^{j+1} ; q\right)_{n}}\right] \\
& =\frac{1}{(1-x q)\left(q^{2} ; q\right)_{\infty}}-\frac{1}{1-x q} \sum_{n \geq 0} \frac{(x q)^{n} q^{n^{2}+n j}}{(q ; q)_{n}\left(q^{2} ; q\right)_{n+j-1}}
\end{aligned}
$$

Note that

$$
\sum_{n \geq 0} \sum_{\lambda \vdash n} x^{\omega(\lambda)} q^{n}=\frac{1}{(1-x q)\left(q^{2} ; q\right)_{\infty}} .
$$

Thus, it follows from (2.12) that

$$
F_{j}(x, q)=\frac{1}{1-x q} \sum_{n \geq 0} \frac{(x q)^{n} q^{n^{2}+n j}}{(q ; q)_{n}\left(q^{2} ; q\right)_{n+j-1}} .
$$

This completes the proof of Theorem 1.3.

Combinatorial Proof. Let us now prove the theorem combinatorially using the concept of Durfee rectangles. Suppose $j \geq 0$. In any partition, the $j$-Durfee rectangle is the largest $n \times(n+j)$ rectangle and the $(-j)$-Durfee rectangle is the largest $(n+j) \times n$ rectangle in its Ferrers diagram. Note that this means that if a partition has $n \times(n+j)$ as its $j$-Durfee rectangle, it has at least $n$ parts, each $\geq n+j$.

For example, if we take the partition $\lambda=(5,4,4,2,2)$ of 17 , we see that it has a $2 \times 4$ rectangle (at least 2 parts $\geq 4$ ) but not a $3 \times 5$ rectangle in its Ferrers diagram. So $2 \times 4$ is the 2 -Durfee rectangle for this partition. In particular, if $j=0$, the Durfee rectangle is the Durfee square of the partition. 
We first consider $F_{-j}(x, q)$ for $j \geq 1$. Take a partition $\lambda$ with $\operatorname{crank}(\lambda) \leq-j$. Since $\operatorname{crank}(\lambda) \leq$ $-j<0, \omega(\lambda) \neq 0$. Indeed if $\omega(\lambda)=0$, then the crank will be the largest part of $\lambda$ by definition, which cannot be negative. Suppose $\lambda$ is not the empty partition. First, note that $\omega(\lambda) \geq j$ because $\operatorname{crank}(\lambda) \leq-j$. Let $n \times(n+j)$ be the $j$-Durfee rectangle in $\lambda$ for some $n \geq 0$. If $n=0$, then the largest part of $\lambda$ is at most $j$, and such $\lambda$ must be generated by

$$
\frac{(x q)^{j}}{(1-x q)\left(q^{2} ; q\right)_{j-1}} \text {. }
$$

If $n \geq 1$, then $\lambda$ has at least $n$ parts, each $\geq n+j$. Suppose $\omega(\lambda)=n+j-i$ for some $i \geq 1$. Then

$$
\mu(\lambda)=(\text { number of parts }>n+j-i) \geq(\text { number of parts } \geq n+j) \geq n .
$$

So

$$
\operatorname{crank}(\lambda)=\mu(\lambda)-\omega(\lambda) \geq n-(n+j-i)=-j+i,
$$

which is a contradiction to the assumption that $\operatorname{crank}(\lambda) \leq-j$. Thus, $\omega(\lambda) \geq n+j$ and $\lambda$ must be generated by

$$
\sum_{n \geq 1} \frac{(x q)^{n+j} q^{n^{2}+n j}}{(q ; q)_{n}(1-x q)\left(q^{2} ; q\right)_{n+j-1}} .
$$

Combining (2.13) and (2.14), we get

$$
F_{-j}(x, q)=\frac{(x q)^{j}}{1-x q} \sum_{n \geq 0} \frac{(x q)^{n} q^{n^{2}+n j}}{(q ; q)_{n}\left(q^{2} ; q\right)_{n+j-1}} .
$$

We note here although the partition of 1 has three different crank values as mentioned in Remark 1.1, only one of them contributes to $F_{-1}(x, q)$ as $x q$, which was taken care of in the case of $n=0$.

For $F_{0}(x, q)$, take a partition $\lambda$. Suppose its Durfee square has size $n \times n$ for some $n \geq 0$. We will consider the following three cases.

- Case 1: $n=0$. then $\lambda$ is the empty partition and its crank is 0 and its contribution to $F_{0}(x ; q)$ is

1.

- Case 2: $n=1$. We divide into two cases: $\lambda$ has no part $>1$ and $\lambda$ has a part $>1$. For the former case, if $\lambda$ is a partition of 1 , then by Remark 1.1, we know that its contribution to $F_{0}(x ; q)$ is

$$
-q+x q
$$

if $\lambda$ is a partition of $N>1$, then it has $N$ parts of size 1 and its crank is $-N$, hence the contribution of such partitions to $F_{0}(x, q)$ is

$$
\frac{(x q)^{2}}{1-x q}
$$

Next, suppose $\lambda$ has a part $>1$. Since $n=1$, i.e., the Durfee square is of size $1 \times 1$, there is indeed only one part $>1$. Thus, if the crank is nonnegative, then $\omega(\lambda) \geq 1$, and the contribution of such partitions to $F_{0}(x, q)$ is

$$
\frac{(x q) q^{2}}{(1-x q)(1-q)} \text {. }
$$


- Case 3: $n \geq 2$. With exactly the same arguments as seen before in $(2.14)$ for $F_{-j}(x, q)$, we can see that such partitions must be generated by

$$
\frac{1}{1-x q} \sum_{n \geq 2} \frac{(x q)^{n} q^{n^{2}}}{(q ; q)_{n}\left(q^{2} ; q\right)_{n-1}} .
$$

By (2.15)-(2.19), we see that

$$
\begin{aligned}
F_{0}(x, q) & =1-q+x q+\frac{(x q)^{2}}{1-x q}+\frac{(x q) q^{2}}{(1-x q)(1-q)}+\frac{1}{1-x q} \sum_{n \geq 2} \frac{(x q)^{n} q^{n^{2}}}{(q ; q)_{n}\left(q^{2} ; q\right)_{n-1}} \\
& =\frac{1-q}{1-x q} \sum_{n \geq 0} \frac{(x q)^{n} q^{n^{2}}}{(q ; q)_{n}^{2}},
\end{aligned}
$$

as desired.

We now prove the statement for $F_{j}(x, q)$ for $j \geq 1$. Take a partition $\lambda$ with crank at most $j$. Suppose $\omega(\lambda)=0$. In this case, the largest part of $\lambda$ is the crank, which is at most $j$. Thus, such $\lambda$ is generated by

$$
\frac{1}{\left(q^{2} ; q\right)_{j-1}} \text {. }
$$

Next, suppose $\omega(\lambda) \neq 0$. Consider the $-j$-Durfee rectangle of $\lambda$ of size $(n+j) \times n$ for some $n \geq 0$. Note that if $(n+j) \times n$ is the $-j$-Durfee rectangle in $\lambda$, it has at least $n+j$ parts, each $\geq n$.

- Case 1: $n \geq 2$. We claim that $\omega(\lambda) \geq n$. Indeed, if $\omega(\lambda)=i$ for some $1 \leq i<n$, then

$$
\mu(\lambda)=(\text { number of parts }>i) \geq(\text { number of parts } \geq n) \geq n+j
$$

and this implies that

$$
\operatorname{crank}(\lambda)=\mu(\lambda)-\omega(\lambda) \geq(n+j)-i>j,
$$

which is a contradiction. Thus, $\omega(\lambda) \geq n$ and $\lambda$ must be generated by

$$
\sum_{n \geq 2} \frac{(x q)^{n} q^{n^{2}+n j}}{(1-x q)\left(q^{2} ; q\right)_{n-1}(q ; q)_{n+j}} .
$$

- Case 2: $n \leq 1$. Since the $-j$-Durfee rectangle is of size at most 1 ,

$$
\nu(\lambda):=(\text { number of parts }>1) \leq j+1 .
$$

Also, since $\omega(\lambda) \geq 1$,

$$
\operatorname{crank}(\lambda)=\mu(\lambda)-\omega(\lambda) \leq \nu(\lambda)-\omega(\lambda) \leq j+1-1 \leq j .
$$

We now further divide into two cases: $\nu(\lambda)=j+1$ and $\nu(\lambda) \leq j$.

- Case 2-1: $\nu(\lambda)=j+1$. In this case, it is clear that $\lambda$ is generated by

$$
\frac{(x q) q^{2(1+j)}}{(1-x q)(q ; q)_{1+j}} \text {. }
$$


- Case 2-2: $\nu(\lambda) \leq j$. Note that partitions with at most $j$ parts $>1$ are generated by

$$
\frac{1}{(q ; q)_{j}}-\frac{q}{(q ; q)_{j-1}}
$$

because the first $q$-product is the generating function for partitions with at most $j$ parts and the second $q$-product is the generating function for partitions with at most $j$ parts and at least one part of size 1 . Thus, $\lambda$ is generated by

$$
\frac{x q}{1-x q}\left(\frac{1}{(q ; q)_{j}}-\frac{q}{(q ; q)_{j-1}}\right)=\frac{x q}{1-x q}\left(\frac{1}{\left(q^{2} ; q\right)_{j-1}}+\frac{q^{j+1}}{(q ; q)_{j}}\right) .
$$

Adding (2.20)-(2.23), we get

$$
\begin{aligned}
F_{j}(x, q) & =\frac{1}{\left(q^{2} ; q\right)_{j-1}}+\frac{x q}{1-x q}\left(\frac{1}{\left(q^{2} ; q\right)_{j-1}}+\frac{q^{j+1}}{(q ; q)_{j}}\right)+\frac{(x q) q^{2(1+j)}}{(1-x q)(q ; q)_{1+j}}+\sum_{n \geq 2} \frac{(x q)^{n} q^{n^{2}+n j}}{(1-x q)\left(q^{2} ; q\right)_{n-1}(q ; q)_{n+j}} \\
& =\frac{1}{1-x q} \sum_{n \geq 0} \frac{(x q)^{n} q^{n^{2}+n j}}{(q ; q)_{n}\left(q^{2} ; q\right)_{n+j-1}} .
\end{aligned}
$$

So we have proved Theorem 1.3 combinatorially.

Remark 2.4. Using Lemma 2.2, we can also verify that for any $j$,

$$
f_{j}(x, q)=F_{j}(x, q)-F_{j-1}(x, q) .
$$

We have the following special case from Theorem 1.3.

Corollary 2.5. We have

$$
\begin{aligned}
\sum_{\operatorname{crank}(\lambda)>0} x^{\omega(\lambda)} q^{|\lambda|} & =\frac{1}{(1-x q)\left(q^{2} ; q\right)_{\infty}}-\frac{1-q}{1-x q} \sum_{n \geq 0} \frac{(x q)^{n} q^{n^{2}}}{(q ; q)_{n}^{2}} \\
& =\sum_{n \geq 1} \frac{\left(1+x q+\cdots+(x q)^{n-1}\right) q^{n^{2}}}{\left(q^{2} ; q\right)_{n-1}(q ; q)_{n}}
\end{aligned}
$$

Proof. The first formula follows immediately from Theorem 1.3. For the second formula, suppose a partition $\lambda$ has its Durfee square of size $n \times n$. Since the number of parts $\geq n$ is at least $n$, if $\omega(\lambda)<n$, then the crank of $\lambda$ would be positive. This proves the second formula.

\section{The $k$-crank generating function weighted by the number of parts in the first subpartition}

We first recall Heine's transformation formula [And98, P. 38]:

$$
\sum_{n \geq 0} \frac{(a ; q)_{n}(b ; q)_{n}}{(q ; q)_{n}(c ; q)_{n}} z^{n}=\frac{(a z ; q)_{\infty}(b ; q)_{\infty}}{(z ; q)_{\infty}(c ; q)_{\infty}} \sum_{n \geq 0} \frac{(c / b ; q)_{n}(z ; q)_{n}}{(q ; q)_{n}(a z ; q)_{n}} b^{n} .
$$

We recall the following result from [LPT21, Lemma 3.1]. Note that we can also obtain this by replacing $z$ by $1 / z$ and $n$ by $n+1$ in Theorem 2.1.

Lemma 3.1. For $k \geq 2$,

$$
\frac{(q ; q)_{\infty}^{2-k}}{(x z q ; q)_{\infty}(q / z ; q)_{\infty}}=\frac{(q ; q)_{\infty}^{1-k}}{(x q ; q)_{\infty}} \sum_{n \geq 0}(-1)^{n} q^{n(n+1) / 2} \frac{(x q ; q)_{n+1}}{(q ; q)_{n}}\left(\frac{1}{1-x z q^{n+1}}+\frac{q^{n+1} / z}{1-q^{n+1} / z}\right)
$$




\section{A. Proof of Theorem 1.4}

In this section, we will prove the formula for the weighted $k$-crank generating function.

Proof. By Lemma 3.1 and the generating function in (1.4), we get

$$
\begin{aligned}
G_{k, d}(x, q) & =\frac{(q ; q)_{\infty}^{1-k}}{(x q ; q)_{\infty}} \sum_{n \geq 0}(-1)^{n} q^{n(n+1) / 2} \frac{(x q ; q)_{n+1}}{(q ; q)_{n}} \frac{\left(x q^{n+1}\right)^{d}}{1-x q^{n+1}} \\
& =\frac{(x q)^{d}(q ; q)_{\infty}^{1-k}}{(x q ; q)_{\infty}} \sum_{n \geq 0}(-1)^{n} q^{n(n+1) / 2} \frac{(x q ; q)_{n}}{(q ; q)_{n}} q^{n d}
\end{aligned}
$$

If we set $a=1 / z, b=x q, c=0, z \rightarrow q^{d+1} z, z \rightarrow 0$ in Heine's transformation formula (3.24), we get

$$
\sum_{n \geq 0}(-1)^{n} q^{n(n+1) / 2} \frac{(x q ; q)_{n}}{(q ; q)_{n}} q^{n d}=(x q ; q)_{\infty}\left(q^{d+1} ; q\right)_{\infty} \sum_{n \geq 0} \frac{(x q)^{n}}{\left(q^{d+1} ; q\right)_{n}(q ; q)_{n}}
$$

so that

$$
\begin{aligned}
G_{k, d}(x, q) & =(q ; q)_{\infty}^{1-k}\left(q^{d+1} ; q\right)_{\infty}(x q)^{d} \sum_{n \geq 0} \frac{(x q)^{n}}{\left(q^{d+1} ; q\right)_{n}(q ; q)_{n}} \\
& =(q ; q)_{\infty}^{2-k} \sum_{n \geq 0} \frac{(x q)^{n+d}}{(q ; q)_{n+d}(q ; q)_{n}}
\end{aligned}
$$

as desired.

Next, observe that

$$
G_{k,-d}(x, q)=\frac{(q ; q)_{\infty}^{1-k}}{(x q ; q)_{\infty}}-\sum_{\operatorname{crank}_{k}(\pi) \leq-d-1} x^{\ell\left(\pi_{1}\right)} q^{|\pi|}
$$

By Lemma 3.1 and the generating function in (1.4), we get

$$
\begin{aligned}
\sum_{\operatorname{crank}_{k}(\pi) \leq-d-1} x^{\ell\left(\pi_{1}\right)} q^{|\pi|} & =\frac{(q ; q)_{\infty}^{1-k}}{(x q ; q)_{\infty}} \sum_{n \geq 0}(-1)^{n} q^{n(n+1) / 2} \frac{(x q ; q)_{n+1}}{(q ; q)_{n}} \frac{\left(q^{n+1}\right)^{d+1}}{1-q^{n+1}} \\
& =\frac{(q ; q)_{\infty}^{1-k}}{(x q ; q)_{\infty}} \sum_{n \geq 1}(-1)^{n-1} q^{n(n+1) / 2} \frac{(x q ; q)_{n}}{(q ; q)_{n}} q^{n d} \\
& =\frac{(q ; q)_{\infty}^{1-k}}{(x q ; q)_{\infty}}\left(1-\sum_{n \geq 0}(-1)^{n} q^{n(n+1) / 2} \frac{(x q ; q)_{n}}{(q ; q)_{n}} q^{n d}\right) \\
& =\frac{(q ; q)_{\infty}^{1-k}}{(x q ; q)_{\infty}}-(q ; q)_{\infty}^{2-k} \sum_{n \geq 0} \frac{(x q)^{n}}{(q ; q)_{n+d}(q ; q)_{n}},
\end{aligned}
$$

where the last equality follows from (3.25). By inserting this into (3.26), we get the second formula.

\section{B. Stacks with summits}

We now consider another type of integer sequences, which appear in the literature under names like stacks, convex compositions, etc. If a sequence $\left(a_{1}, a_{2}, \ldots, a_{r}, c, b_{1}, b_{2}, \ldots, b_{s}\right)$ satisfies

$$
1 \leq a_{1} \leq a_{2} \leq \cdots \leq a_{r} \leq c \geq b_{s} \geq \cdots \geq b_{2} \geq b_{1} \geq 1
$$


then this sequence is said to be unimodal. Such a unimodal sequence is called a stack with summits. The sum of entries and a largest entry are called the size of the stack and a summit, respectively. We note that there may be more than one summit, and to avoid any confusion, the summit $c$ will be marked by a bar put above it. Also, the entries before or after the marked summit $c$ can be empty. For instance, there are six stacks of 3 with summits as follows:

$$
(\overline{3}),(\overline{2}, 1),(1, \overline{2}),(\overline{1}, 1,1),(1, \overline{1}, 1),(1,1, \overline{1}) .
$$

Also, for convenience, we allow the marked summit $c$ to be 0 , and note that if $c=0$, then both sequences $\left(a_{1}, \ldots, a_{r}\right)$ and $\left(b_{1}, \ldots, b_{s}\right)$ are empty.

Let $s s(n)$ be the number of stacks of $n$ with summits for $n \geq 0$. Then it easily follows from the definition that

$$
\sum_{n \geq 0} s s(n) q^{n}=\sum_{n \geq 0} \frac{q^{n}}{(q ; q)_{n}^{2}} .
$$

This generating function can be found in [BrMa14], in which only positive summits are considered.

For an integer $d$, we now generalize stacks with summits as follows:

$$
1 \leq a_{1} \leq a_{2} \leq \cdots \leq a_{r} \leq c \text { and } c-d \geq b_{s} \geq \cdots \geq b_{2} \geq b_{1} \geq 1
$$

with an instance of the summits marked. We call this sequence a $d$-stack with summits. We note here that the sequences $\left(a_{1}, \ldots, a_{r}\right)$ and $\left(b_{1}, \ldots, b_{s}\right)$ may be empty. Also, for $d>0$, the marked summit $c$ must be of size at least $d$ while for $d<0$, the marked summit $c$ can be 0 if $\left(a_{1}, \ldots, a_{r}\right)$ is empty. Then, we can easily see from the definition that

$$
\sum_{\lambda} x^{s(\lambda)} q^{|\lambda|}= \begin{cases}\sum_{n \geq 0} \frac{(x q)^{n+d}}{(q ; q)_{n+d}(q ; q)_{n}}, & \text { if } d \geq 0, \\ \sum_{n \geq 0} \frac{(x q)^{n}}{(q ; q)_{n+|d|}(q ; q)_{n}}, & \text { if } d<0,\end{cases}
$$

where the sum is over all $d$-stacks $\lambda$ with summits and $s(\lambda)$ denotes the size of the marked summit.

By (1.6) and (3.28), we see that the cumulative generating function for 2-colored partitions weighted by the number of parts of the first partition equals the generating function of $d$-stacks with summits weighted by the size of summit. The following theorem encapsulates this result.

Theorem 3.2. The number of 2-colored partitions $\pi$ of $n$ with $\operatorname{crank}_{2}(\pi) \geq d$ and $\ell\left(\pi_{1}\right)=c$ equals the number of d-stacks $\lambda$ of $n$ with summits and $s(\lambda)=c$.

We give a bijective proof of this theorem by conjugation.

Proof. Let $D S S(c, n)$ be the set of $d$-stacks $\lambda$ of $n$ with summit size $c$ and $T(c, n)$ be the set of 2-colored partitions $\pi$ of $n$ with $\operatorname{crank}_{2}(\pi) \geq d$ and $\ell\left(\pi_{1}\right)=c$. We give a bijection from $D S S(c, n)$ to $T(c, n)$.

Suppose $\lambda$ is a $d$-stack $\left(a_{1}, a_{2}, \ldots, a_{r}, c, b_{s}, \ldots, b_{2}, b_{1}\right)$. Remove the bar from $c$ and break $\lambda$ into $\left(c, a_{r}, \ldots, a_{1}\right)$ and $\left(b_{s}, \ldots, b_{1}\right)$. The condition for $d$-stacks in (3.27) ensures that each of $\left(c, a_{r}, \ldots, a_{1}\right)$ and $\left(b_{s}, \ldots, b_{1}\right)$ forms a partition. Let $\pi_{1}$ and $\pi_{2}$ be the conjugates of $\left(c, a_{r}, \ldots, a_{1}\right)$ and $\left(b_{s}, \ldots, b_{1}\right)$, respectively. Then

$$
\ell\left(\pi_{1}\right)=c \geq b_{s}+d=\ell\left(\pi_{2}\right)+d
$$

and so for the 2-color partition $\pi=\left(\pi_{1}, \pi_{2}\right)$,

$$
\operatorname{crank}_{2}(\pi) \geq d .
$$

Also, $\ell\left(\pi_{1}\right)=c=s(\lambda)$ and hence $\pi \in T(c, n)$. This map is clearly bijective and proves Theorem 3.2 combinatorially. 


\section{Conclusions}

Motivated by various questions recently raised by George Beck, we have studied the crank and $k$-crank generating functions weighted by the number of ones and the number of parts in the first subpartitions, respectively, using analytic and combinatorial methods. A natural question arising from our results is: what are the asymptotic behaviors of the functions $F_{j}(x, q)$ and $G_{k, d}(x, q)$. In particular, our results show that the function $F_{j}(x, q)$ is related to Durfee rectangles while the function $G_{k, d}(x, q)$ is related to stacks. The asymptotics for the number of partitions with a given size of the Durfee square or for the number of stacks of a given size can both be found in the literature [BrMa14, CCS98]. Thus, it would be interesting to see if it is possible to use those results to study the asymptotic behaviors of the functions in this paper.

\section{References}

[And98] G.E. Andrews, The Theory of Partitions, Cambridge University Press, Cambridge (1998).

[AnGa88] G.E. Andrews and F. Garvan, Dyson's crank of a partition, Bull. Am. Math. Soc., 18 (1988) $167-171$.

[Ban] S. Bandyopadhyay, Andrews-Beck type congruences related to the crank of a partition, preprint.

[BrMa14] K. Bringmann and K. Mahlburg Asymptotic formulas for stacks and unimodal sequences, Journal of Combinatorial Theory, Series A, 126 (2014) 194-215.

[CCS98] E.R. Canfield, S. Corteel, C.D. Savage Durfee Polynomials, The Electronic Journal of Combinatorics 5.1 (1998) Research paper 32 .

[CCS] S. Chern Weighted partition rank and crank moments. I. Andrews-Beck type congruences, preprint.

[Che21] S. Chern Weighted partition rank and crank moments. II. Odd-order moments, Ramanujan J. (2021).

[Dys44] F.J. Dyson, Some Guesses in the Theory of Partitions, Eureka (Cambridge), 8 (1944) 10-15.

[Fin88] N.J. Fine, Basic Hypergeometric Series and Applications, American Mathematical Society (1988).

[FuTa17] S. Fu and D. Tang On a generalized crank for k-colored partitions, J. Number Theory, 184 (2017) 485 -497.

[GaRa04] G. Gasper and M. Rahman, Basic Hypergeometric Series, 2nd ed., Encyclopedia of Mathematics and its Applications, Cambridge University Press, Cambridge (2004).

[HS20] B. Hopkins and J.A. Sellers, Turning the partition crank, Amer. Math. Monthly, 127 (2020) 654-657.

[HSY22] B. Hopkins, J.A. Sellers and D. Stanton, Dyson's crank and the mex of integer partitions, J. Combin. Theory Ser. A 185 (2022) 105523.

[HSY] B. Hopkins, J. Sellers and A.J. Yee, Combinatorial perspectives on partition crank and mex, preprint.

[HuKi21] J. Huh and B. Kim, On the number of equivalence classes arising from partition involutions II, Discrete Math., 344 (2021) 112410.

[K11] S.J. Kaavya, Crank 0 partitions and the parity of the partition function, Int. J. Number Theory, 7 (3) (2011) $793-801$.

[LPT21] B.L.S. Lin, L. Peng and P.C. Toh, Weighted generalized crank moments for $k$-colored partitions and Andrews-Beck type congruences, Discrete Math., 344 (2021) 112450.

\section{Shreejit Bandyopadhyay \\ Department of Mathematics \\ The Pennsylvania State University \\ University Park, PA 16802, USA \\ e-mail:sxb437@psu.edu}

\section{Ae Ja Yee}

Department of Mathematics

The Pennsylvania State University

University Park, PA 16802, USA

e-mail: yee@psu.edu 